\title{
Persistent Unresolved Inflammation in the Mecp2-308 Female Mutated Mouse Model of Rett Syndrome
}

\author{
Alessio Cortelazzo, ${ }^{1,2,3}$ Claudio De Felice, ${ }^{4}$ Bianca De Filippis, ${ }^{5}$ Laura Ricceri, ${ }^{5}$ \\ Giovanni Laviola, ${ }^{5}$ Silvia Leoncini, ${ }^{1,6}$ Cinzia Signorini, ${ }^{6}$ Monica Pescaglini, ${ }^{3}$ \\ Roberto Guerranti, ${ }^{2,3}$ Anna Maria Timperio, ${ }^{7}$ Lello Zolla, $^{7}$ Lucia Ciccoli, ${ }^{6}$ and Joussef Hayek ${ }^{1}$ \\ ${ }^{1}$ Child Neuropsychiatry Unit, University Hospital Azienda Ospedaliera Universitaria Senese (AOUS), \\ Viale M. Bracci 16, 53100 Siena, Italy \\ ${ }^{2}$ Department of Medical Biotechnologies, University of Siena, Via A. Moro 2, 53100 Siena, Italy \\ ${ }^{3}$ Clinical Pathology Laboratory Unit, University Hospital AOUS, Viale M. Bracci 16, 53100 Siena, Italy \\ ${ }^{4}$ Neonatal Intensive Care Unit, University Hospital AOUS, Viale M. Bracci 16, 53100 Siena, Italy \\ ${ }^{5}$ Centre for Behavioural Sciences and Mental Health, Istituto Superiore di Sanità (ISS), Viale Regina Elena 299, 00161 Rome, Italy \\ ${ }^{6}$ Department of Molecular and Developmental Medicine, University of Siena, Via A. Moro 6, 53100 Siena, Italy \\ ${ }^{7}$ Department of Ecological and Biological Sciences, University of Tuscia, Largo dell'Università, snc, 01100 Viterbo, Italy \\ Correspondence should be addressed to Alessio Cortelazzo; corteale@gmail.com
}

Received 6 December 2016; Accepted 19 April 2017; Published 16 May 2017

Academic Editor: Chiara De Luca

Copyright (c) 2017 Alessio Cortelazzo et al. This is an open access article distributed under the Creative Commons Attribution License, which permits unrestricted use, distribution, and reproduction in any medium, provided the original work is properly cited.

\begin{abstract}
Rett syndrome (RTT) is a rare neurodevelopmental disorder usually caused by mutations in the X-linked gene methyl-CpG-binding protein 2 (MECP2). Several Mecp2 mutant mouse lines have been developed recapitulating part of the clinical features. In particular, Mecp2-308 female heterozygous mice, bearing a truncating mutation, are a validated model of the disease. While recent data suggest a role for inflammation in RTT, little information on the inflammatory status in murine models of the disease is available. Here, we investigated the inflammatory status by proteomic 2-DE/MALDI-ToF/ToF analyses in symptomatic Mecp2-308 female mice. Ten differentially expressed proteins were evidenced in the Mecp2-308 mutated plasma proteome. In particular, 5 positive acute-phase response (APR) proteins increased (i.e., kininogen-1, alpha-fetoprotein, mannose-binding protein C, alpha-1-antitrypsin, and alpha-2-macroglobulin), and 3 negative APR reactants were decreased (i.e., serotransferrin, albumin, and apolipoprotein A1). CD5 antigen-like and vitamin D-binding protein, two proteins strictly related to inflammation, were also changed. These results indicate for the first time a persistent unresolved inflammation of unknown origin in the Mecp2-308 mouse model.
\end{abstract}

\section{Introduction}

Rett syndrome (RTT, MIM 312750) is a progressive neurodevelopmental disorder, affecting almost exclusively the female gender. With a frequency of approximately $1: 10,000$ live births, it is a leading cause of severe intellectual disability and autistic features in females $[1,2]$. The classical clinical picture of the disease [3] is characterized by a period of 6 to 18 months of apparently normal neurodevelopment followed by an early neurological regression, with a progressive loss of acquired cognitive, social, and motor skills in a typical 4-stage neurological regression pattern $[4,5]$. Other known features include stereotypic hand movements, communication dysfunction, seizures, postural hypotonia, tremors, autonomic dysfunction, microcephaly, and growth failure [1]. It has become apparent that there is a spectrum of severity in RTT, as some patients may present with atypical features, sometimes overlapping with autism spectrum disorders [3-5]. RTT is known to be caused in the overwhelming majority of cases by sporadic de novo loss-of-function mutations in the X-linked methyl-CpGbinding protein 2 (MECP2) gene [6] encoding methyl- 
CpG-binding protein 2 (MeCP2), a nuclear protein that binds to methylated $\mathrm{CpGs}$ and regulates gene expression $[7,8]$. Different types of mutations within MECP2 are known to cause RTT, including missense, nonsense, deletions, and insertions [9]. It should be emphasised that most of the proteins identified in mouse serum have orthologs in humans and other mammals [10].

Identification of Mecp2 as the disease-causing gene led rapidly to the development of mouse models of RTT that recapitulate, to varying degrees, the underlying molecular and genetic defects and symptoms of the human disease [11]. Mouse models range from Mecp2-null mutations to specific point mutations mimicking those observed in humans, phenocopying several motor and cognitive features of RTT patients [12-16]. Although mice cannot model all aspects of the human RTT, certainly they recapitulate many features of the disease and are generally accepted as excellent tools to study MeCP2 function [17]. An emerging role for inflammation in human RTT is reported [18-22]. Although $M E C P 2$ is highly expressed in several organs and tissues besides the central nervous system (CNS), including the spleen [16], most of the studies focused on the CNS and little information exists on the inflammatory status in the murine models of the disease, with few exception regarding neuroinflammation processes related to microglia and macrophage function in Mecp2-null mice [23]. Furthermore, prior reports provide a link for $M E C P 2$-RTT to increase erythrocyte sedimentation rate and enhance expression of acute-phase response (APR) proteins and cytokine dysregulation [18-22]. APR is a complex systemic early-defense system activated by trauma, infection, stress, neoplasia, and inflammation [24]. Although nonspecific, it serves as a core of the innate immune response involving physical and molecular barriers and responses that serve to prevent infection, clear potential pathogens, initiate inflammatory processes, and contribute to resolution and the healing process. APR proteins, an integral part of the APR process, have been a focus of many applications in human diagnostic medicine and recently have been identified in common animal species [24]. The number of protein changes was found to be proportional to the severity of the mutation. Our findings revealed for the first time the presence of a subclinical chronic inflammatory status related to the severity carried by the MECP2 gene mutation. Therefore, inflammation seems to be a previously unrecognized feature in RTT, which could play a role in the evolution of the pathology and its severity. Although the genetic mechanisms of RTT have been explored to an extraordinary extent, to date, the details of the biological mechanisms linking the $M E C P 2$ gene mutation to protein expression as a function of clinical phenotype are yet to be clarified.

2-DE/MALDI-ToF/ToF is extremely helpful in order to understand global protein landscape changes in a given biological fluids and/or tissues for a given condition and is considered to be the "gold standard" technique for identifying and characterizing proteins when fitting a series of different parameters, such as peptide matches, sequence coverage (\%), MOWSE score, and $\mathrm{pI} / \mathrm{Mr}(\mathrm{kDa})$ [25]. In particular, with the single exception of two proteomic studies on mouse models [26, 27], very little information exists on possible Mecp2 mouse model-related proteome changes. In the present study, a validated mouse model of RTT was used, that is, the Mecp2-308 mutated model. This model bears a truncating mutation, leading to the expression of a protein truncated at amino acid $308[16,28]$. Several lines of evidence suggest that the truncated Mecp2 protein retains at least some of its functions (i.e., its ability to regulate BDNF gene expression, a Mecp2 target gene) [29], thus contributing to the milder neurobehavioral phenotype of those mice. Based on the previous data demonstrating a clear phenotype in Mecp2-308 females [30], the present study was carried out in symptomatic heterozygous female mice, the genetic and hormonal milieus that, from a translational point of view, is closer to the ones of RTT patients; indeed, among RTT patients, MECP2 mutations are missense and truncated mutations, not deletions $[2,11]$.

The aim of the present study was to investigate in plasma the occurrence of an inflammatory status in the experimental Mecp2-308 mutated Rett female mouse model by using a proteomic 2-DE/MALDI-ToF/ToF approach.

\section{Materials and Methods}

2.1. Animals. The experimental subjects were 10-12-monthold Mecp2-308 heterozygous (Het) female mice [B6.129SMeCP2tm1Hzo/J, stock number: 005439] and wild-type littermates. Mice were housed in polycarbonate transparent cages $(33 \times 13 \times 14 \mathrm{~cm})$ with sawdust bedding and kept on a $12 \mathrm{~h}$ light-dark schedule (lights off at 8:00). Temperature was maintained at $21 \pm 1^{\circ} \mathrm{C}$ and relative humidity at 60 $\pm 10 \%$. Animals were provided ad libitum with a complete pellet diet (Altromin, Germany). All procedures were carried out in accordance with the European Communities Council Directive (10/63/EU) and formally approved by the Italian Ministry of Health.

2.2. Blood Sampling. Blood samples were centrifuged at $2400 \mathrm{~g}$ for $15 \mathrm{~min}$ at $4^{\circ} \mathrm{C}$ and plasma was collected. All the manipulations were carried out within $2 \mathrm{~h}$ after sample collection.

2.3. Electrophoretic Separation of Plasma Proteins after Albumin and IgG Depletion. Albumin and IgG were removed using the ProteoPrep Immunoaffinity Albumin \& IgG Depletion Kit (Sigma-Aldrich), and two-dimensional gel electrophoresis (2-DE) was performed according to Görg et al. [31]. Samples containing $60 \mu \mathrm{g}$ of protein as determined by Bradford [32] were denatured with $10 \mathrm{ml}$ of a solution containing $10 \%$ of SDS and $2.3 \%$ of dithiothreitol (DTT). Afterwards, samples were combined with $350 \mathrm{ml}$ of solubilizing buffer containing $8 \mathrm{M}$ urea, $2 \%$ of 3-[(3-cholamidopropyl)-dimethylammonium]-1-propane sulfonate, $0.3 \%$ DTT, and $2 \%$ of immobilized $\mathrm{pH}$ gradient (IPG) buffer, loaded into $18 \mathrm{~cm}$ IPG strips (pH3-10) nonlinear on an Ettan IPGphor Apparatus system (GE Healthcare), and rehydrated for $7 \mathrm{~h}$. IEF was carried out for a total of $32 \mathrm{kVh}$. After focusing, the strips were equilibrated with the buffer containing $50 \mathrm{mM}$ Tris- $\mathrm{HCl}$ (pH 8.8), $6 \mathrm{M}$ urea, 2\% $w / v$ SDS, 30\% $v / v$ glycerol, and $1 \% w / v$ DTT for $15 \mathrm{~min}$. Subsequently, strips were 
equilibrated again with the same equilibration buffer described above, except that it contained $4 \% w / v$ iodoacetamide instead of DTT and a trace of bromophenol blue. IPG strips and a molecular weight standard were embedded at the top of a $1.5 \mathrm{~mm}$ thick vertical polyacrylamide gradient gel $(8-16 \% \mathrm{~T})$ using $0.5 \% w / v$ agarose and run at a constant current of $40 \mathrm{~mA} /$ gel at $20^{\circ} \mathrm{C}$. The second dimension was performed on an Ettan Daltsix Electrophoresis system (GE Healthcare). Each sample was carried out in triplicate under the same conditions.

2.4. Protein Identification. After mass spectrometrycompatible silver staining, the preparative gel was matched to the master gel in the analytical gel match set [33]. A spot-picking list was generated and exported to Ettan Spot Picker (GE Healthcare). The spots were excised and delivered into 96-well microplates where they were destained and dehydrated with acetonitrile (ACN) for subsequent rehydration with trypsin solution. Tryptic digestion was carried out overnight at $37^{\circ} \mathrm{C}$. Each protein spot digest $(0.75 \mathrm{ml})$ was spotted into the MALDI instrument target and allowed to dry. Then, $0.75 \mathrm{ml}$ of the instrument matrix solution (saturated solution of $\alpha$-cyano-4-hydroxycinnamic acid in 50\% $\mathrm{ACN}$ and $0.5 \% v / v$ trifluoroacetic acid) was applied to dried samples which were dried again. Mass spectra were obtained using an ultrafleXtreme MALDI-ToF/ToF (Bruker Corporation, Billerica, MA, USA), as previously described [34]. After tryptic peptide mass acquisition, mass fingerprint searching was carried out in Swiss-Prot/TREMBL and NCBInr databases using MASCOT (Matrix Science, London, UK, http:// www.matrixscience.com). A mass tolerance of $100 \mathrm{ppm}$ was allowed and only one missed cleavage site was accepted. Alkylation of cysteine by carbamidomethylation was assumed as a fixed modification, whereas oxidation of methionine was considered a possible modification. Criteria used to accept identifications included the extent of sequence coverage, number of matched peptides, and probabilistic scores. Tryptic digests that did not produce MALDI-TOF unambiguous identifications were subjected to ESI-TRAP MS/MS peptide sequencing on a nanospray/LCQ Deca ion trap mass spectrometer (Thermo Finnigan, San Jose, CA, USA).

2.5. Image and Data Analysis. Gel imaging was performed by using ImageMaster 2D Platinum v7.0 software (GE Healthcare). A reference gel for each group was defined for the comparative analyses. The background was subtracted from all gels using the average on-boundary method. Spot volume was expressed as a ratio of the percentage volume $(\% \mathrm{~V})$ detected from the entire gel to minimize differences between samples (i.e., normalization). Unmatched spots or spots with significantly different $\% \mathrm{~V}$ were considered differently expressed. Data were expressed as mean $\pm \mathrm{SD} / \mathrm{SEM}$ or medians [95\% CI for median] as appropriate. Statistical analysis of protein variations was performed using multiple $t$-test with a false discovery rate $(\mathrm{q})$ of 0.05 . Differences between groups were tested using Kruskal-Wallis test or one-way ANOVA, with Dunn's or Holm-Sidak's multiplecomparisons tests for post hoc analyses. A two-tailed $P$ value of less than 0.05 was considered statistically significant. The statistical software GraphPad Prism v6.01 (GraphPad Software Inc., La Jolla, CA, USA) and MedCalc v12.1.4 software package (MedCalc Software, Mariakerke, Belgium) were used.

\section{Results}

3.1. Protein Expression Profile Differences between Mecp2-308 and Wild-Type Mouse Models. Expression changes for a total of 10 differentially expressed proteins were identified in the Mecp2-308 mutated model, relative to wild type, and are shown in 2-DE maps (Figure 1). The proteins were subsequently identified by mass spectrometry. Protein identification as well as peptide matches, sequence coverage, and the probabilistic score was obtained using the MASCOT software (Table 1). A total of 14 spots were identified as follows: serotransferrin (TRFE spots 1 and 2), albumin (ALBU, spot 3 as a fragment), kininogen-1 (KNG1, spot 4), alphafetoprotein (FETA, spot 5), mannose-binding protein C (MBL2, spot 6), apolipoprotein A1 (APOA1, spots 7 and 8), alpha-1-antitrypsin (A1AT, spots 9, 10, and 11), CD5 antigen-like (CD5L, spot 12), alpha-2-macroglobulin (A2M, spot 13), and vitamin D-binding protein (VTDB, spot 14). A two-way analysis of variance showed significant betweensubjects effects (proteins, F: 2157.84, DF: 13, $P<0.001$; mouse category (mutated versus wild type), F: 994.35, DF: $1, P<0.001$; and proteins*mouse category, F: 866.18 , DF: 13, $P<0.001)$.

KNG1, FETA, MBL2, A1AT, and A2M were overexpressed in the examined Mecp2-308 mutated mouse model (Figures 1 and 2, Supplementary material, Supplementary Table 1 available online at https://doi.org/10.1155/2017/ 9467819). In particular, KNG1 and A2M correspond to fragments (KNG1, experimental MW: $12.6 \mathrm{kDa}$; A2M, experimental MW: $64.2 \mathrm{kDa}$ ), both considered products of the entire proteins. Interestingly, all the identified A1AT spots were significantly overexpressed $(P<0.001)$ in the Mecp2308 mutated mouse model.

TRFE, ALBU, and APOA1 were found to be underexpressed in the mutant mouse model. In particular, ALBU was identified as a fragment (experimental MW: $22.6 \mathrm{kDa}$ ), a product of the entire protein (theoretically mapped at MW $70.7 \mathrm{kDa}$ ). Of note, the CD5L spot 12 was overexpressed $(P<0.05)$, while the VTDB spot 14 was significantly underexpressed $(P<0.001)$ in the plasma pattern of the Mecp2308 mutated murine model.

3.2. Biological Functions and APR Role of Differentially Expressed Plasma Proteins in the Mecp2-308 Female Mouse Model. Functions of differentially expressed proteins were evaluated in greater detail specifically for mice with the use of ExPASy, a bioinformatics resource portal operated by the Swiss Institute of Bioinformatics (SIB) (https://www .expasy.org/). The majority of the proteins can be categorized as either positive APR reactants (i.e., KNG1, FETA, MBL2, A1AT, and A2M) or negative APR proteins (i.e., TRFE, ALBU, and APOA1). KNG1, A1AT, and A2M, three protease inhibitors, are strictly related to inflammatory processes and coagulation mechanisms [35-37]. TRFE, 

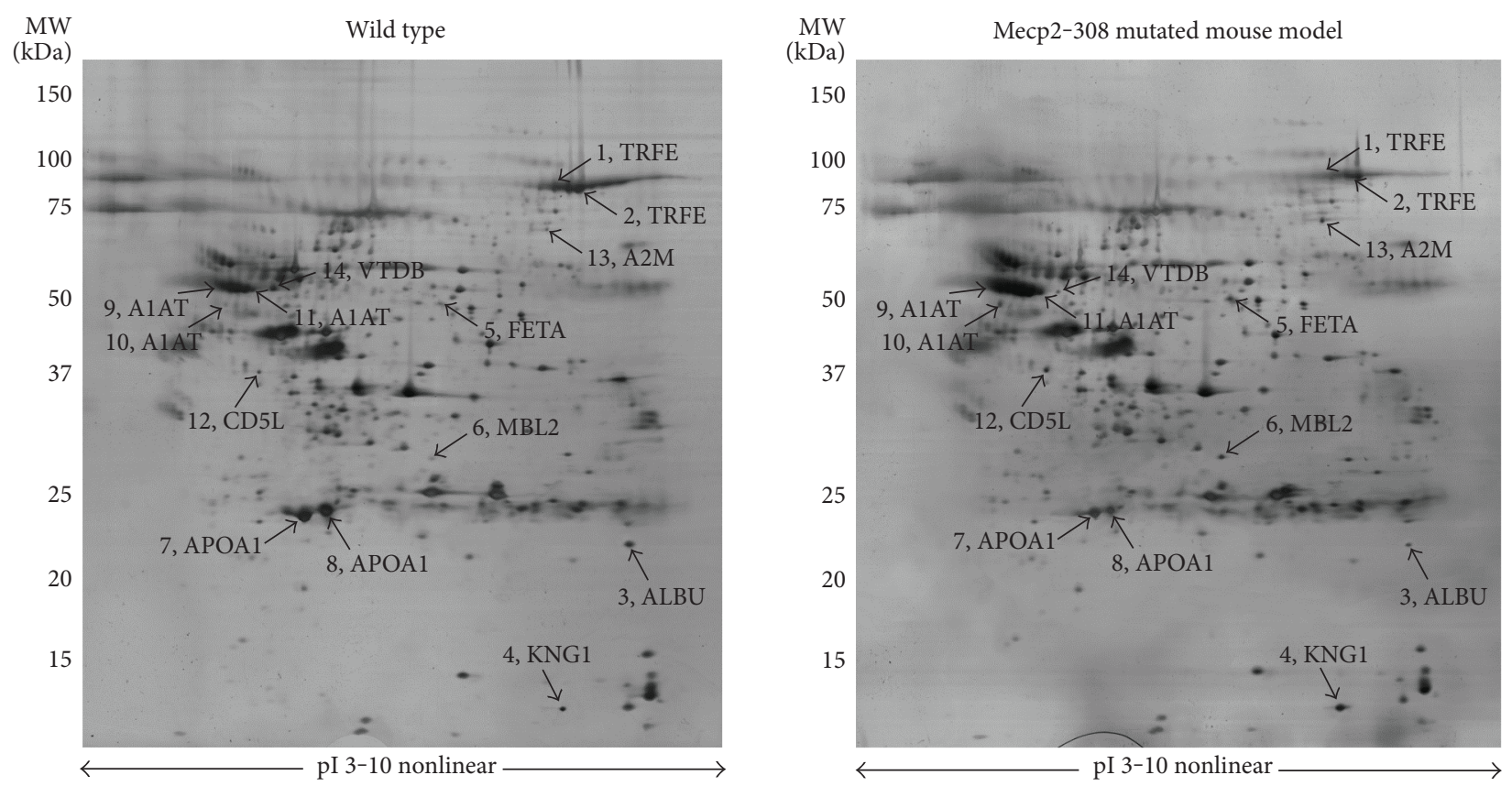

FIGURE 1: Silver-stained 2-DE of proteins from wild-type and Mecp2-308 mutated mouse models. A total of $60 \mu \mathrm{g}$ of protein from albumindepleted plasma samples was subjected to the first dimension electrophoresis on IPG strips, with nonlinear $\mathrm{pH}$ ranging from 3 to 10 (pI, isoelectric point), followed by SDS-polyacrylamide gradient gel (8-16\% T) electrophoresis. Molecular mass (MW, kDa) and pI markers are indicated along the gels. Numbers followed by short names denote the mass spectrometry-identified protein spots listed in Tables 1 and 2.

TABLE 1: Summary of proteins identified in the Mecp2-308 plasma proteome.

\begin{tabular}{|c|c|c|c|c|c|c|c|}
\hline Spot & SwissProt code & Protein name & Short name & Theoretical pI/Mr (kDa) & $\begin{array}{l}\text { Peptide } \\
\text { matches }\end{array}$ & $\begin{array}{c}\text { Sequence } \\
\text { coverage (\%) }\end{array}$ & MOWSE score \\
\hline 1 & Q921I1 & Serotransferrin & TRFE & $6.81 / 79.2$ & $5 / 8$ & 8 & 154 \\
\hline 2 & Q921I1 & Serotransferrin & TRFE & $6.81 / 79.2$ & $9 / 20$ & 16 & 272 \\
\hline 3 & P07724 & Albumin & ALBU & $5.75 / 70.7$ & $17 / 38$ & 26 & 483 \\
\hline 4 & O08677 & Kininogen-1 & KNG1 & $6.05 / 74.1$ & $6 / 9$ & 9 & 219 \\
\hline 5 & P02772 & Alpha-fetoprotein & FETA & $5.47 / 48.7$ & $5 / 8$ & 11 & 148 \\
\hline 6 & P41317 & Mannose-binding protein $\mathrm{C}$ & MBL2 & $4.96 / 26.3$ & $3 / 6$ & 14 & 93 \\
\hline 7 & Q00623 & Apolipoprotein A1 & APOA1 & $5.64 / 30.5$ & $11 / 35$ & 31 & 280 \\
\hline 8 & Q00623 & Apolipoprotein A1 & APOA1 & $5.64 / 30.5$ & $10 / 35$ & 36 & 239 \\
\hline 9 & P07758 & Alpha-1-antitrypsin & A1AT & $5.33 / 46.0$ & $4 / 10$ & 9 & 81 \\
\hline 10 & P07758 & Alpha-1-antitrypsin & A1AT & $5.33 / 46.0$ & $15 / 50$ & 30 & 804 \\
\hline 11 & P07758 & Alpha-1-antitrypsin & A1AT & $5.33 / 46.0$ & $6 / 11$ & 19 & 143 \\
\hline 12 & Q9QWK4 & CD5 antigen-like & CD5L & $5.01 / 40.3$ & $4 / 7$ & 13 & 133 \\
\hline 13 & Q61838 & Alpha-2-macroglobulin & $\mathrm{A} 2 \mathrm{M}$ & $6.27 / 167.0$ & $11 / 25$ & 8 & 370 \\
\hline 14 & P21614 & Vitamin D-binding protein & VTDB & $5.26 / 54.6$ & $14 / 25$ & 7 & 86 \\
\hline
\end{tabular}

Spot numbers match those reported in the representative 2-DE images in Figure 1.

FETA, MBL2, ALBU, APOA1, and VTDB are known to be mainly binding and transport proteins for bioactive molecules and/or inflammatory mediators [38-43]. CD5L is a key regulator of inflammatory response mechanisms and is a macrophage-secreted glycoprotein mainly expressed in lymphoid and inflamed tissues, regulating the tissue macrophage homeostasis [44, 45]. A summary of the biological functions and APR role of the differentially expressed plasma proteins in the Mecp2-308 female model is shown in Table 2.

\section{Discussion}

Our findings indicate for the first time the presence of a persistent unresolved inflammatory status in the Mecp2-308 female mutated mouse model of RTT. The inflammatory 


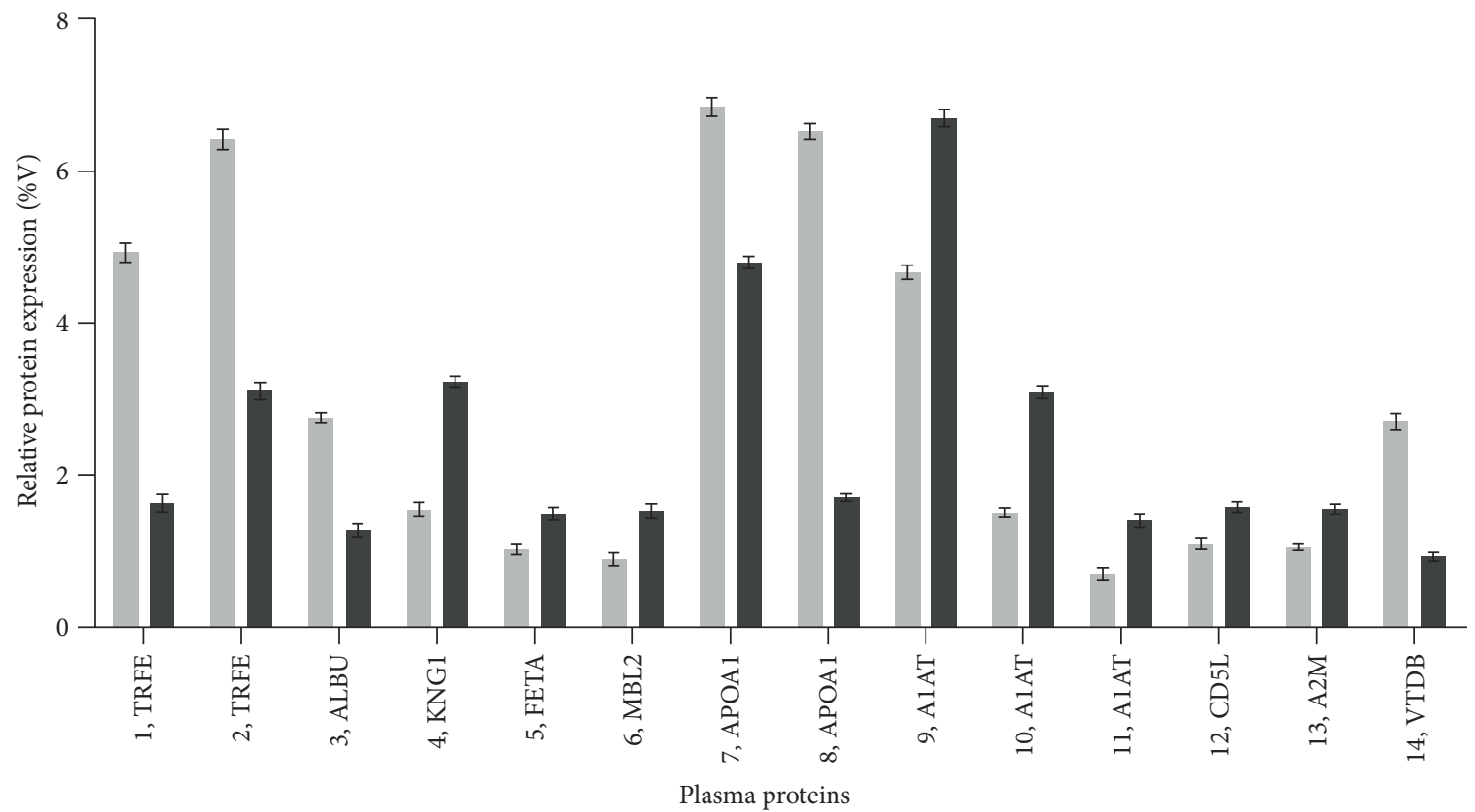

Wild type
Mecp2-308
mutated mouse model

Figure 2: Expression analysis of plasma proteins from wild-type and Mecp2-308 female mutated mouse models. Expression changes of proteins are reported as mean \pm SEM and are statistically significant. Numbers followed by short names refer to proteins reported in Figure 1 .

TABLE 2: Biological functions and APR role of the differentially expressed plasma proteins in the Mecp2-308 female mouse model.

\begin{tabular}{lcccc}
\hline Protein name & Short name & Biological functions (source: ExPASy) & APR proteins \\
\hline Serotransferrin & TRFE & Iron binding and transport & $(-)$ & \\
Albumin & ALBU & Transport and regulation of colloidal osmotic pressure & $(-)$ & $(+)$ \\
Kininogen-1 & KNG1 & Mediator of inflammation and coagulation and protease inhibitor & $(+)$ \\
Alpha-fetoprotein & FETA & Inflammation and fatty acid and metal binding & $(+)$ \\
Mannose-binding protein C & MBL2 & Innate immune defense and inflammation modulation & $(-)$ \\
Apolipoprotein A1 & APOA1 & Lipid transport and metabolism & $(+)$ \\
Alpha-1-antitrypsin & A1AT & Inflammation, coagulation, and protease inhibition & NA \\
CD5 antigen-like & CD5L & Regulation of mechanisms in inflammatory responses & $(+)$ & NA \\
Alpha-2-macroglobulin & A2M & Inflammation, coagulation, and protease inhibition & & \\
Vitamin D-binding protein & VTDB & Vitamin D sterol carrier &
\end{tabular}

APR: acute phase response; (+): positive APR proteins; (-): negative APR proteins; NA: not applicable.

protein pattern was found to be mainly characterized by an attenuated APR, with evident similarities to that of the human disease. The attenuated APR pattern, observed in the Mecp2-308 mutated murine model, shares some relevant features to the protein changes in the plasma protein pattern from RTT girls [18, 46-48]. In particular, plasma from Mecp2-308 mutant mouse shows increased levels of the positive APR reactant A1AT, a multifunctional protein involved in anti-inflammatory and tissue protective properties. More broadly, A1AT plays an important role in modulating immunity, inflammation, apoptosis, and possibly cellular senescence programs [36].
Interestingly, 4 well-known positive APR reactants (i.e., KNG1, FETA, MBL2, and A2M), not previously evidenced in the plasma from RTT patients, specifically characterize the Mecp2-308 inflammatory response. Apart from their typical APR function, these proteins are also involved in the coagulation process (i.e., KNG1 and A2M) [35, 37], fatty acid and metal binding (i.e., FETA) [39], and innate immune defense and inflammatory modulation (i.e., MBL2) [40]. In particular, KNG1 and A2M are two well-known protease inhibitors. KNG1 fragments possess biological activity with moderate to strong correlation with specific diseases (i.e., early progressive renal function decline with type 1 diabetes) 
[35], while those of murine A2M increase during inflammatory responses and tumor growth [37]. Likewise, FETA shows close connections with the modulation of the proinflammatory response of human keratinocytes [39]. Of note, MBL2 comprises a cysteine-rich domain at the $N$-terminus followed by a collagen-like domain, a neck region, and a carbohydrate recognition domain at the $C$-terminus [40]. This domain recognizes and binds to chemical patterns, which include D-mannose, L-fucose, and GlcNAc, carbohydrate residues found on the surface of many pathogens. Furthermore, its structural features suggest a possible role in opsonisation, activating a complement through the lectin complement pathway [40].

A key novel finding for the Mecp2-308 plasma protein pattern is represented by the increase in CD5L coupled with the decrease in VTDB. These two proteins, although not strictly APR reactants, are closely related to inflammatory processes [43-45].

CD5L expression appears to be increased in activated macrophages thus regulating inflammatory response processes $[49,44,45]$. In particular, CD5L is known to prevent Toll-like receptor-induced TNF- $\alpha$ and IL- $1 \beta$ secretion, with a concomitant increase in IL-10 levels, thereby downregulating the tissue macrophage inflammatory reaction [45]. An increased CD5L expression was recently evidenced in plasma from RTT girls in the presence of an inflammatory APR protein response [48]. CD5L induction or upregulation were also reported in response to inflammatory stimuli depending on the variety of physiological situations $[49,50]$. However, the basic mechanism of the CD5L expression induction could depend on a microenvironment (i.e., cell-cell interaction between a specific type of cells and macrophages) [49]. Therefore, also cytokines produced by lymphocytes could contribute to the upregulation of CD5L expression [49]. Interestingly, circulating levels of several cytokines including macrophage-related cytokines such as TNF- $\alpha$, IL-6, IL12 p70, IL-10, TGF- $\beta 1$, IL-8, and RANTES were found to be abnormal in RTT girls [22]. In particular, a strongly increased release of IL-10 was evidenced [22]. The evidenced positive correlation between CD5L and IL-10 suggests a general hyperactivity of the macrophage component of RTT [48]. Several of the observed cytokine pattern changes in MECP2-RTT girls [22] appear to reflect a likely macrophage dysregulation/dysfunction as previously suggested for Mecp2-null mice [19], demonstrating that MeCP2 acts as a regulator of the response to inflammatory stimuli in the microglia and macrophages. Further study will precisely clarify the elements required for CD5L expression induction in RTT. Of note, VTDB levels are reported to be decreased in acute inflammatory conditions [43]. Moreover, VTDB impacts on 25-hydroxyvitamin D levels under different physiologic and pathologic conditions [43]. Furthermore, three negative reactants (i.e., TRFE, ALBU, and APOA1) significantly changed in their expression, in line with our prior study [18]. Alterations in the TRFE levels may lead to abnormal iron metabolism in RTT [38]. Expression of TRFE, previously reported to be involved in autism pathophysiology, decreases during inflammation [38]. Thus, the observed underexpression of TRFE in RTT, as well as in the Mecp2-
308 mutant murine model, would suggest once again that an inflammatory process could play a key role in the pathogenesis of the disease. ALBU is involved in the regulation of the osmotic blood pressure, ions, hormones, and fatty acid binding [41]. Finally, APOA1, a principal component of high-density lipoproteins, shows an impressive portfolio of anti-inflammatory mechanisms at the interface of vascular inflammation and inflamed tissue [42]. Its list of properties includes inhibition of endothelial cell adhesion molecule expression, myeloid lineage cell proliferation, and expression of chemokine receptors and cytokines in synovial lining cells, and neutrophils ingress into inflamed tissues [42].

Very little is known on inflammation and the APR process in Mep2-308 mutated mouse model, as well as in other experimental mice. In Mecp2-308 male mouse brains, an abnormal profile of cytokines has been observed (i.e., IL-6 was dramatically overexpressed, while TNF- $\alpha$ was downregulated), thus suggesting that the morphological deficits of astrocytes are accompanied by aberrant functionality. On the other hand, no data are available on plasma cytokine levels in this Mecp2 mouse line. Recently, high levels of TNF- $\alpha$, IL-6, and IL-3 have been reported in an in vitro human peripheral blood mononuclear cell (PBMC) experimental setting in which the MECP2 gene was silenced [21].

Clinical studies support the presence of differences in the clinical manifestation of the syndrome in RTT patients carrying different mutations in the MECP2 gene. From a translational point of view, this is consistent with the profound differences in the symptom gravity and in the age of their onset exhibited by the various mouse models developed. Indeed, different RTT models are characterized by different genetic mutations and by consequent different degrees of protein integrity [17]. We have chosen to study the Mecp2308 RTT mouse model since among RTT patients, MECP2 mutations are missense and truncated mutations, so this model more closely resembles the clinical phenotype shown by the majority of the patients compared to null models. In particular, the Mecp2-308 model is characterized by a delayed onset of symptoms and a prolonged life-span $[16,28]$ in comparison with models in which the gene is deleted. Importantly, the behavioral phenotype of Mecp2-308 recapitulates most of the clinical phenotype shown by RTT patients carrying C-terminal deletions of the MECP2 gene [51].

Reduction in several plasma proteins, potentially affecting cholesterol transport and inhibiting oxidation phenomena, is known to occur during inflammation. These proteins include cholesterol ester transfer protein, hepatic lipase, and apolipoproteins. It is thought that reduction in these proteins, associated with an increase in positive APR proteins, may change the high-density lipoprotein from anti-inflammatory into proinflammatory particles [52].

A recent report, analyzing the response of astrocytes during activation by proinflammatory stimulation, proposes that the protective phenotype against iron-mediated oxidative stress in cell death involves a complex change in the expression and activity of several genes involved in the control of the cellular redox state [53]. Therefore, it is plausible that abnormal redox status and unrecognized 
proinflammatory stimuli would converge in RTT patients to functionally damage the astrocytes within the CNS. There is a mounting evidence for a persistent redox imbalance in RTT [54-56]. This abnormal redox homeostasis could be related to the wider context of an unresolved inflammatory process whose fine mechanisms remain to be elucidated.

Inflammation plays an important role in the pathophysiology of several common diseases, such as Alzheimer's and atherosclerosis. Thus, the cellular mechanisms by which resolution occurs and the key biochemical pathways associated with the return to homeostasis/catabasis (return from disease) clearly open many new avenues for potential therapeutic interventions in a wide range of diseases associated with unresolved inflammation [57].

RTT appears to be more complex than previously thought. A gene sequence analysis has indicated several hundreds of gene mutations associated with the MECP2 gene mutation and, therefore, to be considered potential disease modifiers [58].

MECP2 levels start to increase postnatally with the protein being abundant in the mature nervous system [59]. MeCP2 protein expression is not uniform in different neuronal cell populations and parts of the brain [60] and is known to change with age [61]. MeCP2 is a multifunctional protein influencing gene expression and metabolism on many levels [62]. MeCP2 is currently considered a global, genome-wide transcriptional regulator (i.e., mainly repressor) whose function is well correlated with DNA methylation density for a large proportion of unusually long brain-specific genes [62]. Likely, a loss of MeCP2 function causes a transcriptional imbalance leading to a suboptimal brain homeostasis [63]. A clear cut proof-ofconcept demonstration for the effects of $\mathrm{MeCP} 2$ gene mutations on the homeostasis of the brain is the absence of major phenotypes associated with Rett-like mice when levels of the central nervous system MeCP2 are normal [64]. MECP2 dampens neuronal transcription globally, thus allowing activity-related responses essential for the learning process and specific synapsis formation $[65,66]$.

Although the genetic mechanisms of RTT have been explored to an extraordinary extent, to date the details of the biological mechanisms linking the $M E C P 2$ gene mutation to protein expression as a function of clinical phenotype have yet to be clarified. The findings of the present study add a further phenotypical feature, thus expanding our knowledge on the molecular cascade of events that lead to the disease phenotype.

\section{Conclusion}

Our results indicate for the first time the occurrence of a persistent unresolved inflammation of unknown origin in the Mecp2-308 female mouse model of RTT, which appears to be helpful to elucidate in greater detail the importance of the inflammatory status, disentangle the role of inflammation in RTT on the phenotype, and contribute in developing inflammation-targeted therapeutic strategies.

\section{Abbreviations}

A1AT: Alpha-1-antitrypsin

A2M: Alpha-2-macroglobulin

ALBU: Albumin

APOA1: Apolipoprotein A1

CD5L: CD5 antigen-like

FETA: Alpha-fetoprotein

KNG1: Kininogen-1

MBL2: Mannose-binding protein C

TRFE: Serotransferrin

VTDB: Vitamin D-binding protein.

\section{Conflicts of Interest}

The authors declare that they have no conflict of interest.

\section{Authors' Contributions}

Alessio Cortelazzo and Claudio De Felice contributed equally to this work.

\section{Acknowledgments}

The authors heartily thank the internationally recognized illustrator Roberto Innocenti (http://www.robertoinnocenti .com) for the continued support and the sensitization work towards Rett syndrome and Dr. Roberto Faleri (Central Medical Library) for the online bibliographic assistance. This research was supported by grant from the Jerome Lejeune Foundation (France) and AIRETT (Italy) to Giovanni Laviola and by the IRSF HeART Award (no.3107) to Bianca De Filippis. Special thanks go to Rett girls and their families who represent the ultimate goal of our research.

\section{References}

[1] M. Chahrour and H. Y. Zoghbi, "The story of Rett syndrome: from clinic to neurobiology," Neuron, vol. 56, no. 3, pp. 422437, 2007.

[2] L. S. Weaving, C. J. Ellaway, J. Gecz, and J. Christodoulou, "Rett syndrome: clinical review and genetic update," Journal of Medical Genetics, vol. 42, no. 1, pp. 1-7, 2005.

[3] A. Rett, "On a unusual brain atrophy syndrome in hyperammonemia in childhood," Wiener Medizinische Wochenschrift, vol. 116, no. 37, pp. 723-726, 1966.

[4] B. Hagberg, "Clinical manifestations and stages of Rett syndrome," Mental Retardation and Developmental Disabilities Research Reviews, vol. 8, no. 2, pp. 61-65, 2002.

[5] J. L. Neul, W. E. Kaufmann, D. G. Glaze et al., "Rett syndrome: revised diagnostic criteria and nomenclature," Annals of Neurology, vol. 68, no. 6, pp. 944-950, 2010.

[6] R. E. Amir, I. B. Van den Veyver, M. Wan, C. Q. Tran, U. Francke, and H. Y. Zoghbi, "Rett syndrome is caused by mutations in X-linked MECP2, encoding methyl-CpG binding protein 2," Nature Genetics, vol. 23, no. 2, pp. 185-188, 1999.

[7] M. Chahrour, S. Y. Jung, C. Shaw et al., "MeCP2, a key contributor to neurological disease, activates and represses transcription," Science, vol. 320, no. 5880, pp. 1224-1229, 2008. 
[8] P. L. Jones, G. J. Veenstra, P. A. Wade et al., "Methylated DNA and MeCP2 recruit histone deacetylase to repress transcription," Nature Genetics, vol. 19, no. 2, pp. 187191, 1998.

[9] T. Bienvenu and J. Chelly, "Molecular genetics of Rett syndrome: when DNA methylation goes unrecognized," Nature Reviews. Genetics, vol. 7, no. 6, pp. 415-426, 2006.

[10] R. Wait, G. Chiesa, C. Parolini et al., "Reference maps of mouse serum acute-phase proteins: changes with LPS-induced inflammation and apolipoprotein A-I and A-II transgenes," Proteomics, vol. 5, no. 16, pp. 4245-4253, 2005.

[11] D. M. Katz, J. E. Berger-Sweeney, J. H. Eubanks et al., "Preclinical research in Rett syndrome: setting the foundation for translational success," Disease Models \& Mechanisms, vol. 5, no. 6, pp. 733-745, 2012.

[12] R. Z. Chen, S. Akbarian, M. Tudor, and R. Jaenisch, "Deficiency of methyl-CpG binding protein-2 in CNS neurons results in a Rett-like phenotype in mice," Nature Genetics, vol. 27, no. 3, pp. 327-331, 2001.

[13] P. Moretti, J. A. Bouwknecht, R. Teague, R. Paylor, and H. Y. Zoghbi, "Abnormalities of social interactions and homecage behavior in a mouse model of Rett syndrome," Human Molecular Genetics, vol. 14, no. 2, pp. 205-220, 2005.

[14] J. D. Picker, R. Yang, L. Ricceri, and J. Berger-Sweeney, “An altered neonatal behavioral phenotype in Mecp2 mutant mice," Neuroreport, vol. 17, no. 5, pp. 541-544, 2006.

[15] M. Santos, A. Silva-Fernandes, P. Oliveira, N. Sousa, and P. Maciel, "Evidence for abnormal early development in a mouse model of Rett syndrome," Genes, Brain, and Behavior, vol. 6, no. 3, pp. 277-286, 2007.

[16] M. Shahbazian, J. Young, L. Yuva-Paylor et al., "Mice with truncated $\mathrm{MeCP} 2$ recapitulate many Rett syndrome features and display hyperacetylation of histone H3," Neuron, vol. 35, no. 2, pp. 243-254, 2002.

[17] L. Ricceri, B. De Filippis, and G. Laviola, "Mouse models of Rett syndrome: from behavioural phenotyping to preclinical evaluation of new therapeutic approaches," Behavioural Pharmacology, vol. 19, no. 5-6, pp. 501-517, 2008.

[18] A. Cortelazzo, C. De Felice, R. Guerranti et al., "Subclinical inflammatory status in Rett syndrome," Mediators of Inflammation, vol. 2014, Article ID 480980, 13 pages, 2014.

[19] J. C. Cronk, N. C. Derecki, E. Ji et al., "Methyl-CpG binding protein 2 regulates microglia and macrophage gene expression in response to inflammatory stimuli," Immunity, vol. 42, no. 2, pp. 679-691, 2015.

[20] N. C. Derecki, E. Privman, and J. Kipnis, "Rett syndrome and other autism spectrum disorders-brain diseases of immune malfunction?" Molecular Psychiatry, vol. 15, no. 4, pp. 355363,2010

[21] C. M. O’Driscoll, M. P. Lima, W. E. Kaufmann, and J. P. Bressler, "Methyl CpG binding protein 2 deficiency enhances expression of inflammatory cytokines by sustaining NF- $\kappa \mathrm{B}$ signaling in myeloid derived cells," Journal of Neuroimmunology, vol. 283, pp. 23-29, 2015.

[22] S. Leoncini, C. De Felice, C. Signorini et al., "Cytokine dysregulation in MECP2- and CDKL5-related Rett syndrome: relationships with aberrant redox homeostasis, inflammation, and $\omega$-3 PUFAs," Oxidative Medicine and Cellular Longevity, vol. 2015, Article ID 421624, 18 pages, 2015.

[23] M. Horiuchi, L. Smith, I. Maezawa, and L. W. Jin, "CX(3)CR1 ablation ameliorates motor and respiratory dysfunctions and improves survival of a Rett syndrome mouse model," Brain, Behavior, and Immunity, vol. 60, pp. 106-116, 2017.

[24] C. Cray, J. Zaias, and N. H. Altman, "Acute phase response in animals: a review," Comparative Medicine, vol. 59, no. 6, pp. 517-526, 2009.

[25] N. L. Anderson and N. G. Anderson, "The human plasma proteome: history, character, and diagnostic prospects," Molecular and Cellular Proteomics, vol. 2, no. 1, p. 50, 2003.

[26] V. Matarazzo and G. V. Ronnett, "Temporal and regional differences in the olfactory proteome as a consequence of MeCP2 deficiency," Proceedings of the National Academy of Sciences of the United States of America, vol. 101, no. 20, pp. 7763-7768, 2004.

[27] A. Cortelazzo, C. De Felice, R. Guerranti et al., "Abnormal Nglycosylation pattern for brain nucleotide pyrophosphatase-5 (NPP-5) in Mecp2-mutant murine models of Rett syndrome," Neuroscience Research, vol. 105, pp. 28-34, 2016.

[28] B. De Filippis, L. Ricceri, and G. Laviola, "Early postnatal behavioral changes in the Mecp2-308 truncation mouse model of Rett syndrome," Genes, Brain, and Behavior, vol. 9, no. 2, pp. 213-223, 2010.

[29] L. Lyon, P. W. Burnet, J. N. Kew et al., "Fractionation of spatial memory in GRM2/3 (mGlu2/mGlu3) double knockout mice reveals a role for group II metabotropic glutamate receptors at the interface between arousal and cognition," Neuropsychopharmacology, vol. 36, no. 13, pp. 2616-2628, 2011.

[30] B. De Filippis, M. Musto, L. Altabella, E. Romano, R. Canese, and G. Laviola, "Deficient purposeful use of forepaws in female mice modelling Rett syndrome," Neural Plasticity, vol. 2015, Article ID 326184, 13 pages, 2015.

[31] A. Görg, C. Obermaier, G. Boguth et al., "The current state of two-dimensional electrophoresis with immobilized $\mathrm{pH}$ gradients," Electrophoresis, vol. 21, no. 6, pp. 1037-1053, 2000.

[32] M. M. Bradford, "A rapid and sensitive method for the quantitation of microgram quantities of protein utilizing the principle of protein-dye binding," Analytical Biochemistry, vol. 72, no. 1-2, pp. 248-254, 1976.

[33] E. Mortz, T. N. Krogh, H. Vorum, and A. Görg, "Improved silver staining protocols for high sensitivity protein identification using matrix-assisted laser desorption/ionization-time of flight analysis," Proteomics, vol. 1, no. 11, pp. 1359-1363, 2001.

[34] U. Hellman, C. Wernstedt, J. Góñez, and C. H. Heldin, "Improvement of an "In-Gel" digestion procedure for the micropreparation of internal protein fragments for amino acid sequencing," Analytical Biochemistry, vol. 224, no. 1, pp. 451455, 1995.

[35] I. Isordia-Salas, R. A. Pixley, I. M. Sáinz, C. Martínez-Murillo, and R. W. Colman, "The role of plasma high molecular weight kininogen in experimental intestinal and systemic inflammation," Archives of Medical Research, vol. 35, no. 5, pp. 369377, 2004.

[36] J. M. Hunt and R. Tuder, "Alpha 1 anti-trypsin: one protein, many functions," Current Molecular Medicine, vol. 12, no. 7, pp. 827-835, 2012.

[37] T. Jinbo, T. Sakamoto, and S. Yamamoto, "Serum $\alpha 2$ macroglobulin and cytokine measurements in an acute inflammation model in rats," Laboratory Animals, vol. 36, no. 2, pp. 153-157, 2002.

[38] D. A. Loeffler, J. R. Connor, P. L. Juneau et al., "Transferrin and iron in normal Alzheimer's disease, and Parkinson's 
disease brain regions," Journal of Neurochemistry, vol. 65, no. 2, pp. 710-716, 1995.

[39] G. J. Mizejewski, “Alpha-fetoprotein (AFP) and inflammation: is AFP an acute and/or chronic phase reactant?" Journal of Hematology \& Thromboembolic Diseases, vol. 3, no. 1, pp. 19, 2015.

[40] M. M. Dean, R. M. Minchinton, S. Heatley, and D. P. Eisen, "Mannose binding lectin acute phase activity in patients with severe infection," Journal of Clinical Immunology, vol. 25, no. 4, pp. 346-352, 2005.

[41] J. P. Nicholson, M. R. Wolmarans, and G. R. Park, "The role of albumin in critical illness," British Journal of Anaesthesia, vol. 85, no. 4, pp. 599-610, 2000.

[42] D. Nguyen, M. Nickel, C. Mizuguchi, H. Saito, S. Lund-Katz, and M. C. Phillips, "Interactions of apolipoprotein A-I with high-density lipoprotein particles," Biochemistry, vol. 52, no. 11, pp. 1963-1972, 2013.

[43] P. Yousefzadeh, S. A. Shapses, and X. Wang, "Vitamin D binding protein impact on 25-Hydroxyvitamin D levels under different physiologic and pathologic conditions," International Journal of Endocrinology, vol. 2014, Article ID 981581, 6 pages, 2014.

[44] L. Sanjurjo, N. Amézaga, C. Vilaplana et al., "The scavenger protein apoptosis inhibitor of macrophages (AIM) potentiates the antimicrobial response against Mycobacterium tuberculosis by enhancing autophagy," PloS One, vol. 8, no. 11, article e79670, 2013.

[45] L. Sanjurjo, N. Amézaga, G. Aran et al., "The human CD5L/ AIM-CD36 axis: a novel autophagy inducer in macrophages that modulates inflammatory responses," Autophagy, vol. 11, no. 3, pp. 487-502, 2015.

[46] A. Cortelazzo, R. Guerranti, C. De Felice et al., "A plasma proteomic approach in Rett syndrome: classical versus preserved speech variant," Mediators of Inflammation, vol. 2013, Article ID 438653, 10 pages, 2013.

[47] C. De Felice, A. Cortelazzo, C. Signorini et al., "Effects of $\omega$-3 polyunsaturated fatty acids on plasma proteome in Rett syndrome," Mediators of Inflammation, vol. 2013, Article ID 723269, 9 pages, 2013.

[48] A. Cortelazzo, C. De Felice, S. Leoncini et al., "Inflammatory protein response in CDKL5-Rett syndrome: evidence of a subclinical smouldering inflammation," Inflammation Research, vol. 66, no. 3, pp. 269-280, 2017.

[49] I. Haruta, Y. Kato, E. Hashimoto et al., "Association of AIM, a novel apoptosis inhibitory factor, with hepatitis via supporting macrophage survival and enhancing phagocytotic function of macrophages," The Journal of Biological Chemistry, vol. 276, no. 25, pp. 22910-22914, 2001.

[50] L. Sanjurjo, G. Aran, N. Roher, A. F. Valledor, and M. R. Sarrias, "AIM/CD5L: a key protein in the control of immune homeostasis and inflammatory disease," Journal of Leukocyte Biology, vol. 98, no. 2, pp. 173-184, 2015.

[51] S. Díaz de León-Guerrero, G. Pedraza-Alva, and L. PérezMartinez, "In sickness and in health: the role of methylCpG binding protein 2 in the central nervous system," The European Journal of Neuroscience, vol. 33, no. 9, pp. 1563-1574, 2011.

[52] B. J. Van Lenten, S. T. Reddy, M. Navab, and A. M. Fogelman, "Understanding changes in high density lipoproteins during the acute phase response," Arteriosclerosis, Thrombosis, and Vascular Biology, vol. 26, no. 8, pp. 1687-1688, 2006.
[53] R. Macco, I. Pelizzoni, A. Consonni et al., "Astrocytes acquire resistance to iron-dependent oxidative stress upon proinflammatory activation," Journal of Neuroinflammation, vol. 10, no. 1, p. 130, 2013.

[54] C. De Felice, C. Signorini, S. Leoncini et al., "The role of oxidative stress in Rett syndrome: an overview," Annals of the new York Academy of Sciences, vol. 1259, pp. 121-135, 2012.

[55] C. Signorini, C. De Felice, T. Durand et al., "Isoprostanes and 4-hydroxy-2-nonenal: markers or mediators of disease? Focus on Rett syndrome as a model of autism spectrum disorder," Oxidative Medicine and Cellular Longevity, vol. 2013, Article ID 343824, 10 pages, 2013.

[56] C. De Felice, F. Della Ragione, C. Signorini et al., "Oxidative brain damage in Mecp2-mutant murine models of Rett syndrome," Neurobiology of Disease, vol. 68, pp. 66-77, 2014.

[57] C. N. Serhan, S. D. Brain, C. D. Buckley et al., "Resolution of inflammation: state of the art, definitions and terms," FASEB Journal, vol. 21, no. 2, pp. 325-332, 2007.

[58] E. Grillo, C. Lo Rizzo, L. Bianciardi et al., "Revealing the complexity of a monogenic disease: Rett syndrome exome sequencing," PloS One, vol. 8, no. 2, article e56599, 2013.

[59] N. Kishi and J. D. Macklis, "MECP2 is progressively expressed in post-migratory neurons and is involved in neuronal maturation rather than cell fate decisions," Molecular and Cellular Neurosciences, vol. 27, no. 3, pp. 306-321, 2004.

[60] K. Sugino, C. M. Hempel, B. W. Okaty et al., "Cell-type-specific repression by methyl-CpG-binding protein 2 is biased toward long genes," The Journal of Neuroscience, vol. 34, no. 38, pp. 12877-12883, 2014.

[61] D. Jain, K. Singh, S. Chirumamilla et al., "Ocular MECP2 protein expression in patients with and without Rett syndrome," Pediatric Neurology, vol. 43, no. 1, pp. 35-40, 2010.

[62] M. J. Lyst and A. Bird, "Rett syndrome: a complex disorder with simple roots," Nature Reviews. Genetics, vol. 16, no. 5, pp. 261-275, 2015.

[63] R. Ruth and A. P. Bird, "MeCP2 mutations: progress towards understanding and treating Rett syndrome," Genome Medicine, vol. 9, no. 1, p. 7, 2017.

[64] P. D. Ross, J. Guy, J. Selfridge et al., "Exclusive expression of $\mathrm{MeCP} 2$ in the nervous system distinguishes between brain and peripheral Rett syndrome-like phenotypes," Human Molecular Genetics, vol. 25, no. 20, pp. 4389-4404, 2016.

[65] L. M. Monteggia and E. T. Kavalali, "Rett syndrome and the impact of MeCP2 associated transcriptional mechanisms on neurotransmission," Biological Psychiatry, vol. 65, no. 3, pp. 204-210, 2009.

[66] F. Ehrhart, S. L. Coort, E. Cirillo, E. Smeets, C. T. Evelo, and L. M. Curfs, "Rett syndrome - biological pathways leading from MECP2 to disorder phenotypes," Orphanet Journal of Rare Diseases, vol. 11, no. 1, p. 158, 2016. 


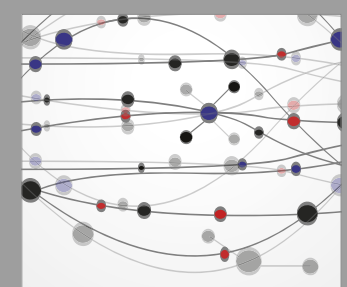

The Scientific World Journal
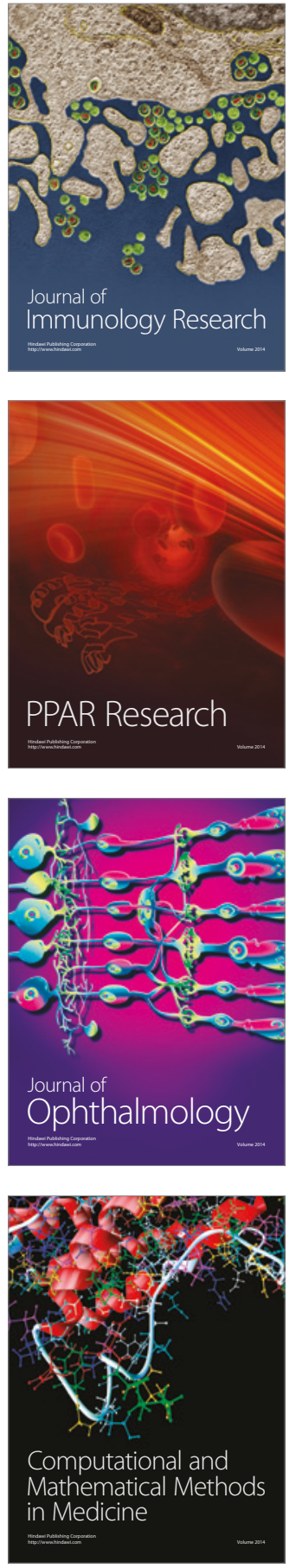

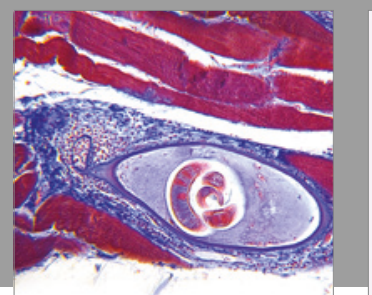

Gastroenterology Research and Practice
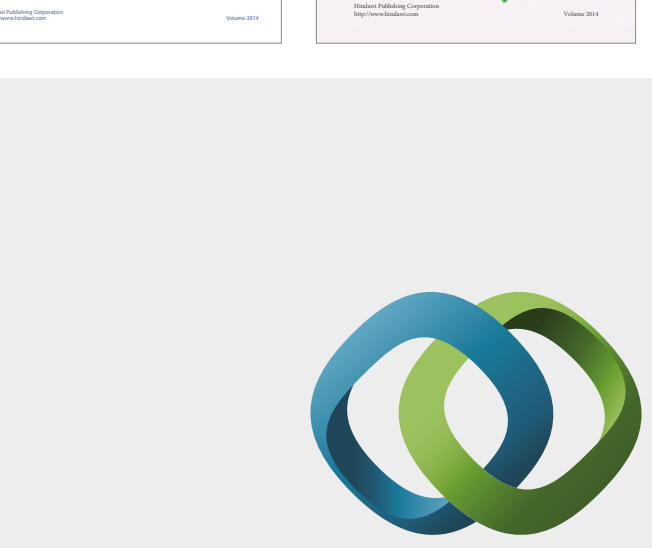

\section{Hindawi}

Submit your manuscripts at

https://www.hindawi.com
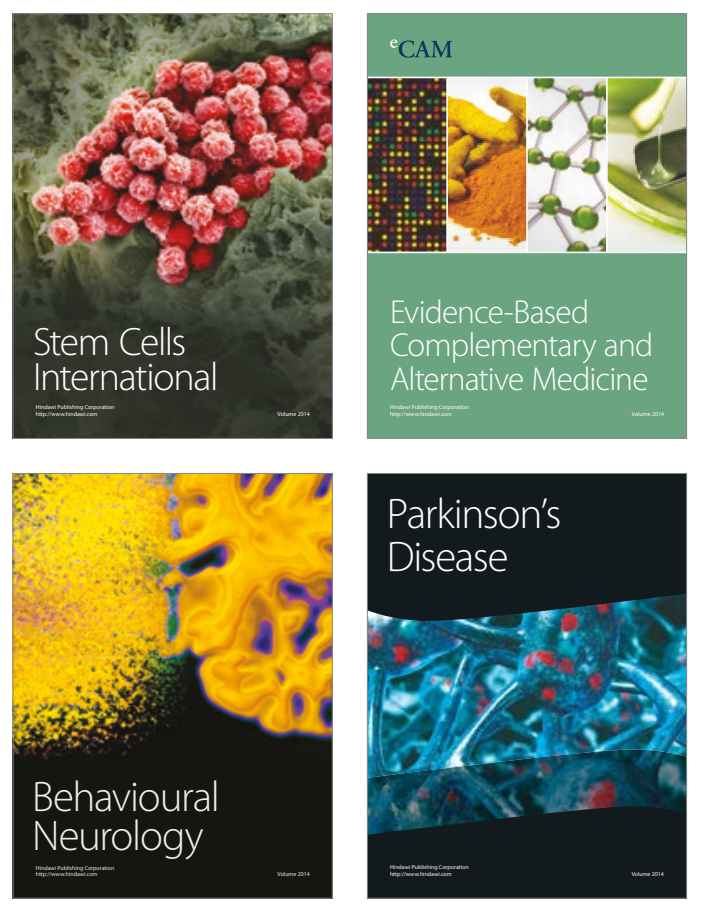
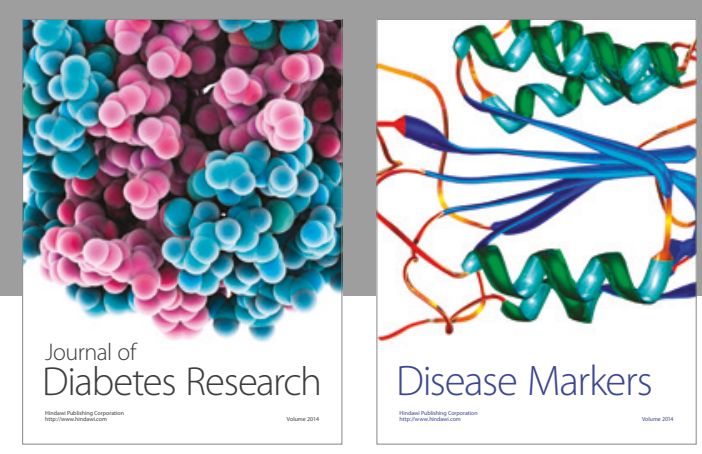

Disease Markers
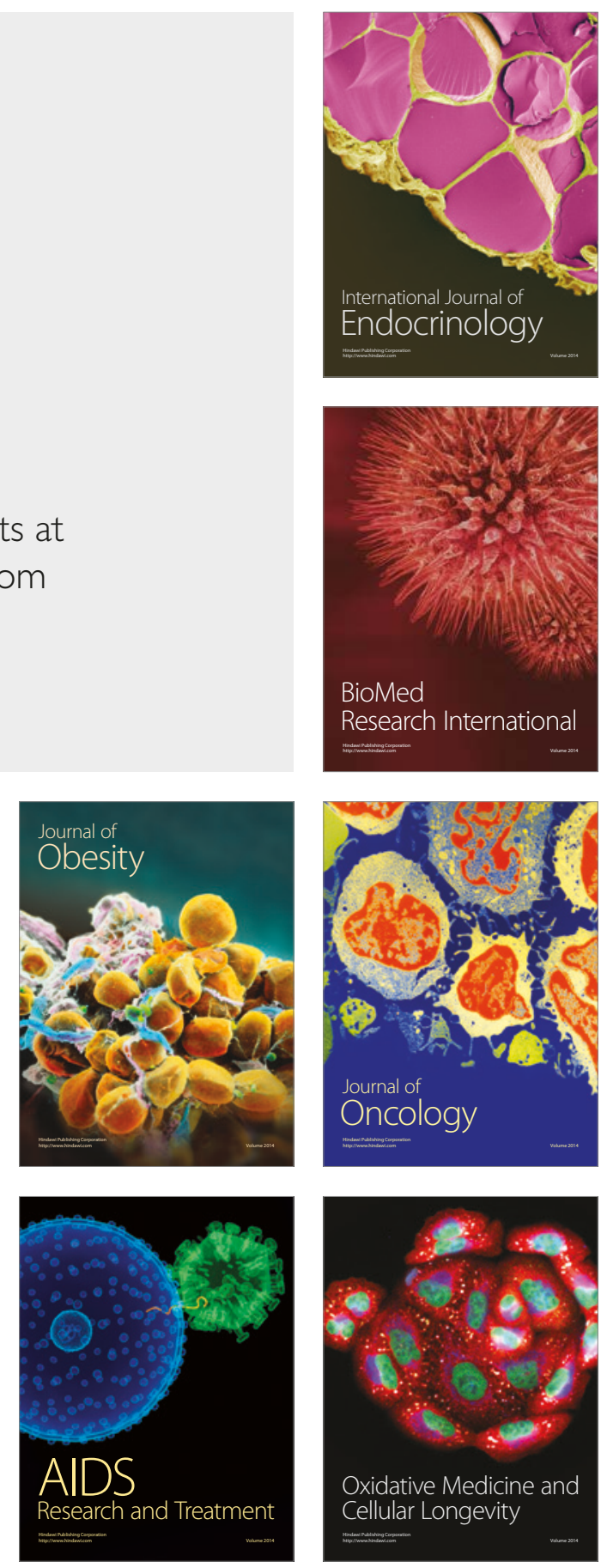